15. Craciun E, Rosulescu I. On Danubian endemic familial nephropathy (Balkan nephropathy). American Journal of Medicine 1970; 49: 774-9.

16. Toncheva D, Dimitrov T. Genetic predisposition to Balkan endemic nephropathy. Nephron 1996; 72: 564-9.
17. Mandal AK. Sindjic M, Sommers SC. Kidney pathology in endemic nephropathy. Clinical Nephrology 1987; 27: 304-8.

18. Ferluga D, Hvala A, Vizjak A, Trnacevic S, Halilbašic A. Renal function, protein excretion, and pathology of Balkan endemic nephropathy. III. Light and electron microscopic studies. Kidney International 1991; 40 (Sup): S57-67.

\title{
Trends and determinants of childhood stunting and underweight in Sri Lanka
}

\author{
R P Rannan-Eliya ${ }^{1}$, S M M Hossain ${ }^{2}$, C Anuranga ${ }^{1}$, R Wickramasinghe $^{1}$, R Jayatissa ${ }^{3}$, A T P L Abeykoon ${ }^{3}$
}

(Index words: stunting, wasting, inequality, children, maternal height, WHO growth standard)

\begin{abstract}
Introduction Child undernutrition is a major risk factor for child mortality and adult ill-health. Despite substantial progress in most health indicators, undernutrition remains high in Sri Lanka, with recent trends being unclear, owing to methodological differences in national surveys.

Methods This study uses data from the 1987, 1993, 2000 and 2006-07 Demographic and Health Surveys (DHS) and the 2009 Nutrition and Food Security Survey (NFSS) to investigate trends and determinants of child undernutrition in Sri Lanka. The prevalence rates of stunting, underweight and wasting were re-estimated using the 2006 WHO growth standards to ensure consistency. Multivariate regression analysis was then undertaken to analyse the determinants of height-forage in children aged 9-23 months, and 24-59 months, and the relative impact of key factors was assessed using prediction models.

Results Stunting and wasting substantially improved from 1987 to 2000, but rates stagnated from 2000 to 2006/07. Whilst economic inequalities in under nutrition were greater than in most other countries, the multivariate analysis found that maternal height, household wealth, length of breast-feeding and altitude are significant determinants of stunting, but differences in child feeding practices and other factors were not. Of these, maternal height and household wealth had the most influence.

Conclusion The results are consistent with the finding that food insecurity is the main driver of undernutrition, but more research is required to validate this. The strong relationship of child height with maternal height suggests
\end{abstract}

that epigenetic factors, proxied by short maternal height, constrain the applicability of the WHO growth standards in Sri Lanka.

Ceylon Medical Journal 2013; 58: 10-18

\section{Introduction}

Childhood under nutrition, manifesting as stunting, underweight and wasting, remains a substantial problem in Sri Lanka. Long-term sequelae include reduced cognitive ability and school performance, lower economic productivity, and decreased offspring birth weight [1]. Children who are undernourished in the first two years and put on weight rapidly later are at higher risk of chronic diseases, such as diabetes and ischaemic heart disease, both of which are increasing in Sri Lanka. Recent surveys have reported deteriorating trends in child malnutrition, but the published statistics lack comparability [2-5]. Our study re-examines the data to estimate trends and disparities, and investigate determinants of stunting.

\section{Methods}

We used data from all relevant, recent, national surveys: the DHS 1987, 1993, 2000 and 2006-07 surveys of the Department of Census and Statistics and the Medical Research Institute's NFSS 2009 [7] [2-5]. The DHS surveys exclude the Eastern (EP) and Northern (NP) Provinces, except for the1987 and 2006-07 surveys, which partially or completely covered EP. The NFSS 2009 used a two-stage, cluster sample design to survey 13 districts

${ }^{1}$ Institute for Health Policy, Colombo, Sri Lanka, ${ }^{2}$ UNICEF, Colombo, Sri Lanka and ${ }^{3}$ Medical Research Institute, Colombo, Sri Lanka.

Correspondence: RPRE, e-mail: <raviofficelk@gmail.com>. Received 18 June and revised version accepted 11 October 2012. Competing interests: Views expressed by authors in this article are not necessarily those of UNICEF. 
from all nine provinces, but this design does not support reliable national inferences.

These surveys collected data on the height and weight of all children, born to mothers aged 15-49 years, of age 0 59 months (DHS 2006/07, NFSS 2009), 3-59 months (DHS 1993 and 2000) and 3-36 months (DHS 1987). This allows the computation of the deviation in $\mathrm{z}$ scores of a child from the median of a reference population in terms of height for age (HAZ), weight for age (WAZ) and weight for height (WHZ). As per convention, stunting is defined as $\mathrm{HAZ}<2.0$, wasting as $\mathrm{WAZ}<2.0$, and underweight as $\mathrm{WHZ}<2.0$.

To allow analysis of living standards, household wealth indices were constructed, based on household assets, using Principle Components Analysis (PCA), and households ranked into sample-weighted wealth quintiles [8]. The DHS 2006/07 report tabulated nutrition indicators by quintiles, but we report this for the first time for all DHS surveys. Variables were also constructed for the altitude of individual households using their GPS coordinates and for the level of mother's decision-making autonomy, using methods previously described [9]. For the NFSS, altitude was imputed as the average for the Grama Niladhari area.

Statistical analyses were carried out using Stata, version 12.0 (StataCorp, College Station, TX, USA), taking into account the multistage cluster sampling design of the surveys. During regression analyses, potential variable interactions were tested for, and multicollinearity of variables was assessed, using variance inflation factors.

\section{Results}

\section{Trends in child undernutrition}

The DHS 1987, 1993 and 2000 published statistics use the NCHS/WHO growth reference, which is based on a study of US children, whilst the DHS 2006/07 and NFSS 2009 used the newer 2006 WHO child growth standards, which are based on the WHO Multicentre Growth Reference Study (MGRS), involving exclusively breastfed children from six different regions of the world [10-11]. The WHO 2006 standards produce higher estimates of stunting and wasting at all ages, and higher rates of underweight during 0-6 months, and lower thereafter. The differences in growth reference, age groups, geographic coverage and sampling design render trend comparisons using the published statistics invalid. To obtain comparable trends, we reanalysed all the surveys, excluding EP and NP, and consistently applied the WHO 2006 standard. Table 1 presents estimates of undernutrition from each survey, with aggregate estimates provided for comparable age groups of children aged 3-36, 3-59 and 0-59 months to facilitate comparison.

Stunting and underweight fell substantially from 1987 to 2000 , but there was little change from 2000 to $2006 / 07$, whilst wasting showed no real trend. The differences in prevalence between the two last surveys were only significant at $5 \%$ for wasting (survey-adjusted Wald tests for stunting $p=0.19$, for under weight $p=0.08$ and for wasting, $\mathrm{p}=0.04$ for children aged 3-59 months). The NFSS 2009 estimates for undernutrition are higher than the DHS $2007 / 07$ estimates, but they are not comparable, owing to differences in sampling design. Separate analysis indicates that its sample comprises poorer households than those in the DHS survey, which would lead to a systematic upward bias in its estimates.

Figure 1 compares the age trajectories in mean HAZ, WAZ and WHZ scores in 1993 and 2006/07. HAZ starts close to standard in 1993, but growth falters substantially from 3 months to 30 months, declining at $0.06 z$ scores per month, before stabilizing and then gradually recovering. In 2006/07, growth falters at a slower rate and stabilizes earlier by 24 months. WAZ starts with a substantial deficit, consistent with the high incidence of low birth weight, but subsequent trends in WAZ scores are similar to HAZ, confirming that the most critical period for growth faltering in Sri Lanka is the first two years of life, comparable to trends in most developing countries [12]. WHZ starts below the standard, and then falls steadily through the first 59 months, although the rate of decline reduces between the two surveys.

\section{Disparities in undernutrition}

Consistent inequalities in stunting and underweight are observed in all the surveys, details of which are separately published [13]. Stunting and underweight are higher in male and multiple births, and decrease with maternal education and body mass index (BMI). Both are higher and increasingly concentrated in the central hill country, higher in rural than urban areas, and highest in estate areas, but the urban-rural disparity is narrowing. Stunting and underweight are substantially higher in all surveys in Indian Tamils. In the most recent surveys, prevalence in Sri Lankan Tamils has been modestly aboveaverage, but less in Muslims than other ethnic groups. In contrast, but consistent with global patterns, disparities in wasting are small and have not changed significantly [14].

Stunting and underweight increase with impoverishment of the family, as proxied by the wealth index (Table 2). Prevalence rates in the poorest quintile are three-times that in the richest quintile (DHS 2006/07 - stunting: 29\% versus $8 \%$, and underweight: $31 \%$ versus $11 \%$ ). The wealth-associated inequalities in the NFSS data are smaller, but the wealth index for the NFSS incorporates fewer variables than for the DHS. This makes it a weaker proxy measure, which would yield a lower estimate of any socioeconomic gradient.

Wealth inequalities were quantified using the Concentration Index (CI) [9, 15]. The estimated CI values reveal substantial inequality in undernutrition, being negative and highly significant $(p<0.001)$, and changing little over time. In comparison with a WHO-supported study of countries from all regions of the world, the degree of inequality in stunting in Sri Lanka in 2006/07 is greater than in 41 out of 48 countries, with the only countries with greater inequality being from Latin America, Nigeria and Turkey [14]. 
Table 1. Child undernutrition rates by age group according to WHO growth standard, DHS and NFSS surveys 1987-2009

\begin{tabular}{|c|c|c|c|c|c|c|}
\hline \multirow[t]{2}{*}{ Age in months } & \multicolumn{3}{|c|}{ DHS } & \multicolumn{3}{|c|}{ NFSS 2009} \\
\hline & 1987 & 1993 & 2000 & $2006 / 07$ & $\begin{array}{c}\text { Excluding } \\
E P \text { \& NP }\end{array}$ & $\begin{array}{l}\text { Including } \\
E P \& N P\end{array}$ \\
\hline \multicolumn{7}{|l|}{ Stunting } \\
\hline $3-11$ & 19.1 & 14.2 & 7.2 & 11.5 & 13.8 & 12.5 \\
\hline $12-23$ & 34.3 & 29.0 & 19.8 & 19.2 & 20.2 & 19.9 \\
\hline $24-35$ & 36.4 & 33.6 & 24.3 & 20.5 & 20.5 & 21.0 \\
\hline $36-59$ & $*$ & 31.3 & 19.7 & 16.1 & 21.4 & 20.8 \\
\hline Total 3-35 & 31.1 & 26.4 & 17.6 & 17.5 & 18.6 & 18.3 \\
\hline Total 3-59 & $*$ & 28.5 & 18.5 & 17.0 & 19.6 & 19.3 \\
\hline Total 0-59 & $*$ & $*$ & $*$ & 16.7 & 19.6 & 19.1 \\
\hline \multicolumn{7}{|l|}{ Underweight } \\
\hline $3-11$ & 19.0 & 15.0 & 13.0 & 13.8 & 13.8 & 13.0 \\
\hline $12-23$ & 28.6 & 22.8 & 19.0 & 20.1 & 19.6 & 19.0 \\
\hline $24-35$ & 37.0 & 32.2 & 27.1 & 22.6 & 27.0 & 26.7 \\
\hline $36-59$ & * & 38.1 & 27.8 & 24.1 & 27.5 & 27.5 \\
\hline Total 3-35 & 29.1 & 23.7 & 20.1 & 19.2 & 20.8 & 20.2 \\
\hline Total 3-59 & $*$ & 29.7 & 23.4 & 21.2 & 23.3 & 23.0 \\
\hline Total 0-59 & $*$ & $*$ & $*$ & 20.9 & 23.2 & 22.8 \\
\hline \multicolumn{7}{|l|}{ Wasting } \\
\hline $3-11$ & 8.7 & 13.3 & 13.5 & 10.8 & 9.0 & 8.9 \\
\hline $12-23$ & 14.2 & 9.0 & 14.6 & 13.7 & 10.4 & 10.3 \\
\hline $24-35$ & 15.6 & 14.8 & 15.0 & 13.7 & 14.1 & 14.3 \\
\hline $36-59$ & $*$ & 19.0 & 18.9 & 16.0 & 14.1 & 14.3 \\
\hline Total 3-35 & 13.3 & 12.1 & 14.4 & 12.9 & 11.4 & 11.4 \\
\hline Total 3-59 & $*$ & 15.0 & 16.3 & 14.2 & 11.4 & 12.5 \\
\hline Total 0-59 & $*$ & $*$ & $*$ & 14.2 & 12.5 & 12.5 \\
\hline $\mathbf{N}$ & 1,977 & 3,166 & 2,196 & 6,012 & 2,381 & 3,822 \\
\hline
\end{tabular}

* Age groups not sampled in survey.
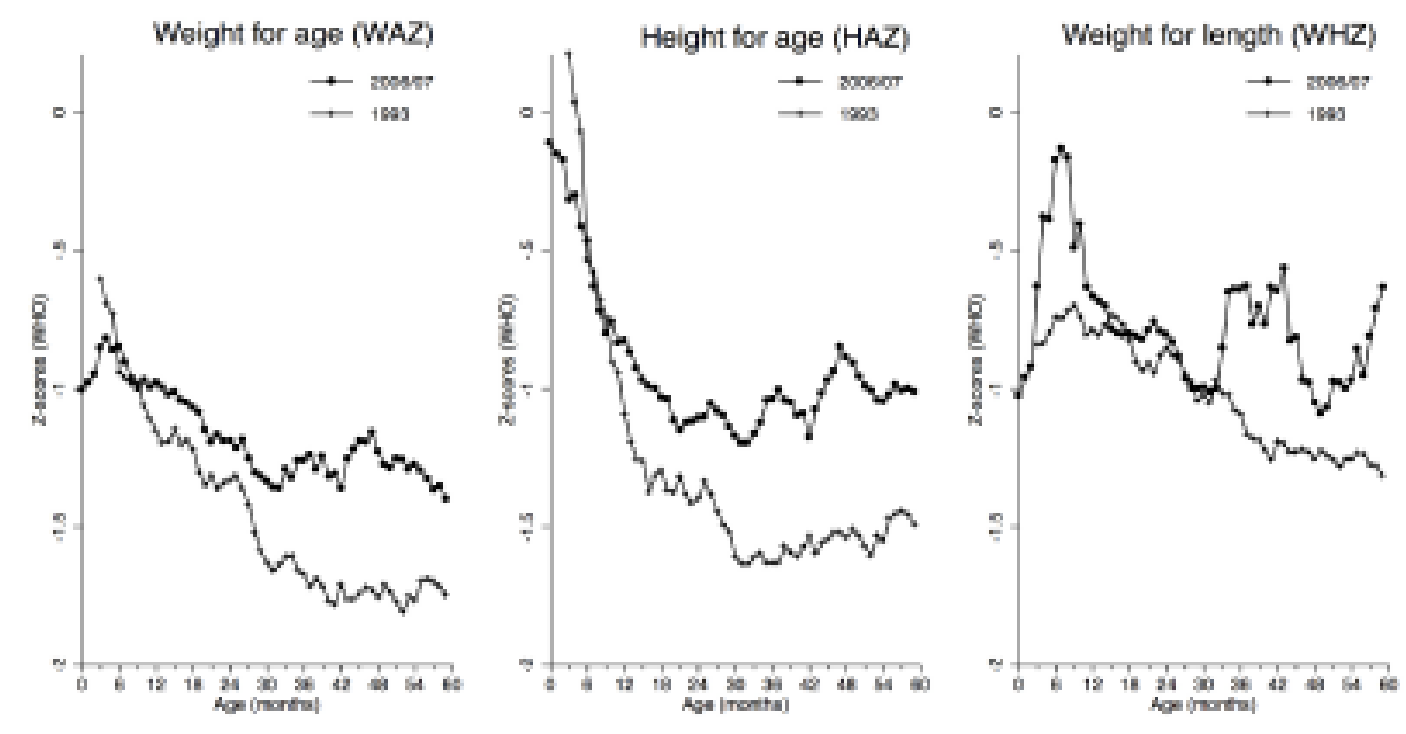

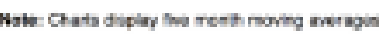

Figure 1. Mean anthropometric scores by age (0-59 months), Sri Lanka DHS 1993 and 2006-07. 
Table 2. Inequalities in stunting and underweight in children aged less than 5 years, DHS and NFSS surveys 1987-2009

\begin{tabular}{|c|c|c|c|c|c|c|}
\hline & \multicolumn{5}{|c|}{ Prevalence of stunting by wealth quintile } & \multirow{2}{*}{ Concentration Index } \\
\hline & Poorest & Q2 & Q3 & Q4 & Richest & \\
\hline \multicolumn{7}{|c|}{ Stunting (3-35 months) } \\
\hline DHS 1987 & 40.3 & 39.2 & 37.3 & 23.5 & 15.2 & $-0.17 * * *$ \\
\hline DHS 1993 & 39.0 & 34.1 & 23.1 & 23.3 & 10.9 & $-0.21 * * *$ \\
\hline DHS 2000 & 30.1 & 18.7 & 13.3 & 7.8 & 3.5 & $-0.38 * * *$ \\
\hline DHS 2006/07 & 28.3 & 22.1 & 15.7 & 14.8 & 8.9 & $-0.21 * * *$ \\
\hline NFSS 2009 & 23.0 & 19.9 & 15.9 & 16.2 & 10.8 & $-0.12 * * *$ \\
\hline \multicolumn{7}{|c|}{ Underweight (3-35 months) } \\
\hline DHS 1987 & 41.3 & 36.0 & 27.6 & 23.5 & 15.5 & $-0.18 * * *$ \\
\hline DHS 1993 & 32.9 & 31.8 & 22.9 & 18.3 & 10.9 & $-0.20 * * *$ \\
\hline DHS 2000 & 31.5 & 19.4 & 20.7 & 9.2 & 8.6 & $-0.26 * * *$ \\
\hline DHS $2006 / 07$ & 27.4 & 25.6 & 17.7 & 16.8 & 9.3 & $-0.19 * * *$ \\
\hline NFSS 2009 & 23.7 & 21.8 & 18.7 & 21.9 & 11.9 & $-0.11 * * *$ \\
\hline \multicolumn{7}{|c|}{ Stunting (0-59 months) } \\
\hline DHS $2006 / 07$ & 28.8 & 21.9 & 15.9 & 13.4 & 8.4 & $-0.23 * * *$ \\
\hline \multicolumn{7}{|c|}{ Underweight (0-59 months) } \\
\hline DHS 2006/07 & 30.4 & 25.6 & 22.3 & 18.1 & 11.4 & $-0.18 * * *$ \\
\hline
\end{tabular}

${ }^{1}$ Significant at $* * * p<.001, * * p<.01, * p<.05$

\section{Regression analysis}

We then investigated the determinants of height-forage (HAZ) in the DHS 2006/07 and NFSS 2009 using multivariate linear regression, as reduced HAZ is the best indicator of chronic undernutrition. The NFSS data set is smaller than the DHS data set, but it includes a larger set of feeding indicators. Given the different trajectories of $\mathrm{HAZ}$ during the first two years and later, separate analyses were undertaken for children aged 9-23 months and 24-59 months of age. The nine month cut-off allows use of the recom-mended international indicators for assessing infant and young child feeding (IYCF) practices, which gauge breast feeding $(\mathrm{BF})$ and complementary feeding $[16]$.

Selection of potential covariates was based on a literature review and the UNICEF framework for determinants of childhood malnutrition [17]. They were grouped into infant and birth factors, maternal characteristics, IYCF practices, household food security, household resources and characteristics and geographical location.

Model building was done in a stepwise additive manner, considering statistical significance and plausibility. Covariates that were dropped for reasons of no significant impact were teenage pregnancy, multiple birth, vitamin A supplementation, child illness in past two weeks (diarrhoea, fever, respiratory symptoms), mother currently in employment, access to safe water, household size, tsunami-affected neighbourhood, parity, preceding birth interval, maternal illness (taking medication for asthma, hypertension or heart disease), maternal decision-making autonomy and paternal education. The latter five were available in the DHS only.

Table 3 and 4 present the final model estimates of the determinants of HAZ, based on analysis of the two data sets, but we focus on the analysis of the larger DHS data set. The impacts of several factors differ in the two growth periods, validating the decision to model each separately. The decrease in HAZ with child age during the first two years is statistically significant, but the subsequent increase with age is not statistically significant $(p=0.26)$. Birth weight has a substantial, although diminishing, impact on attained height, with every $100 \mathrm{~g}$ increase in birth weight associated with a 0.04-0.07 increase in HAZ scores by the fifth year. Female children are smaller initially, but this disparity disappears later. Not being the firstborn child and increasing numbers of children in the family are associated with reduced growth, which might be due to competition for family resources. Altitude has a highly significant, negative impact on HAZ, equivalent to a 0.03 0.04 reduction in HAZ scores with every 100 metres increase in elevation. Its impact is consistent with studies elsewhere, and can be physiologically explained by the exponential decline of partial oxygen pressure with altitude and its impact on haemoglobin saturation [18]. 


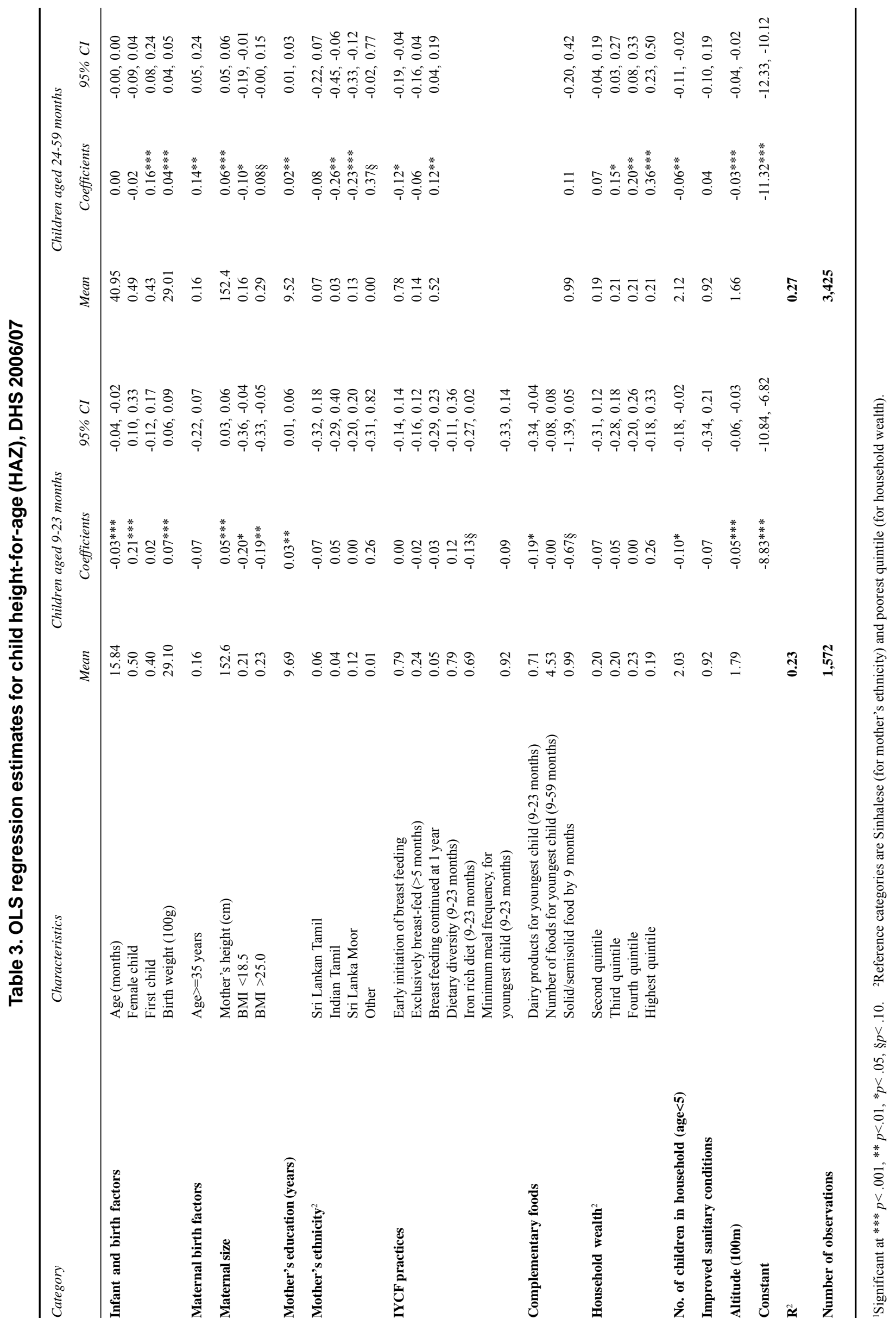




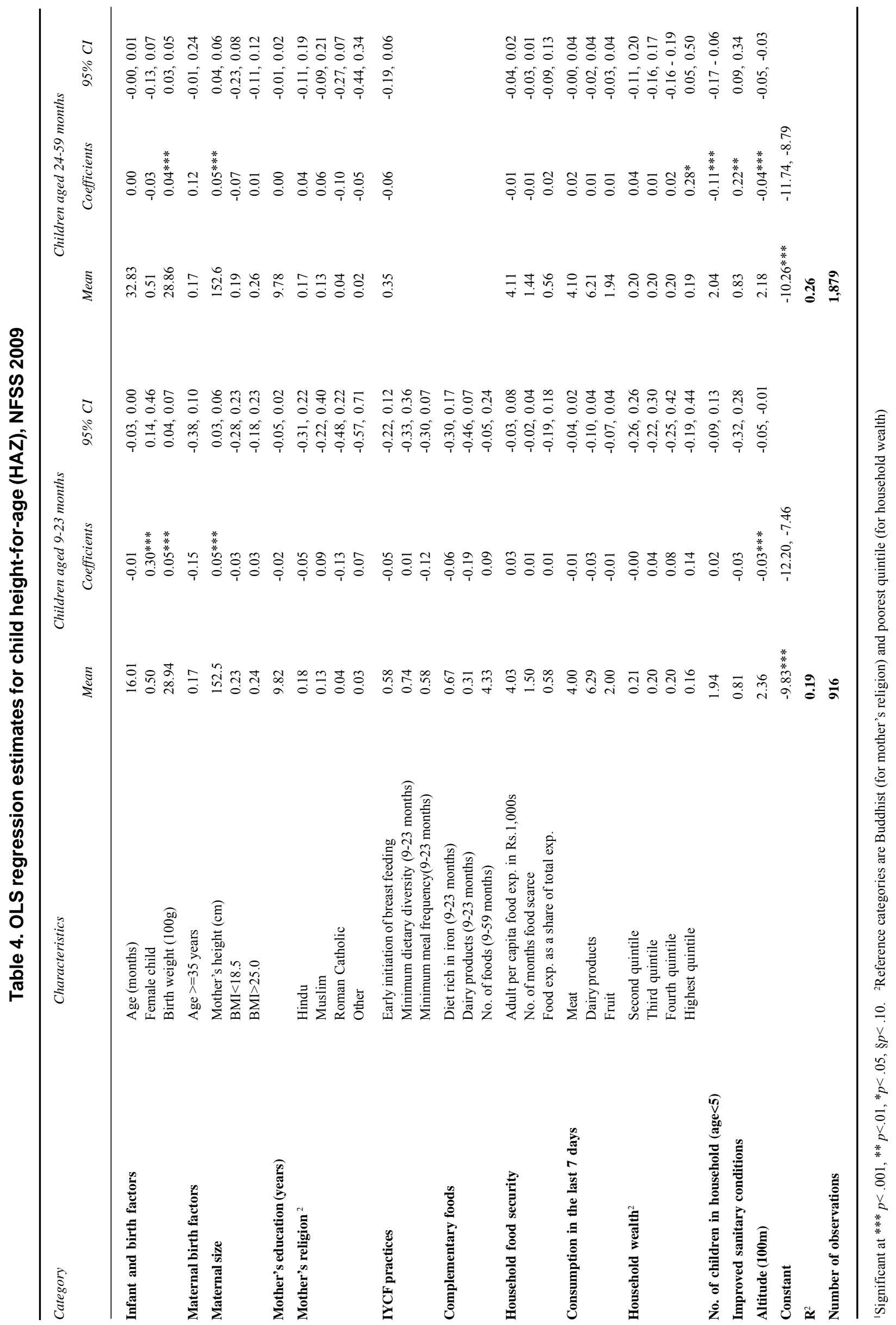


Maternal weight (both under and over), has a negative impact in the first two years, but only maternal underweight is associated with reduced HAZ in subsequent years. In contrast, maternal height has a large and consistent positive impact on HAZ scores throughout, equivalent to a 0.03-0.05 increase in HAZ scores for every $1 \mathrm{~cm}$ increase in height. There is also a positive association between increased maternal education and HAZ, whilst being an older mother has a positive association after two years.

In both data sets, we fail to find a consistent or significant relationship between most IYCF indicators and HAZ and, between the available household food security indicators, and HAZ in the NFSS data set. The only exception is continued breastfeeding for at least 12 months, which has a significant positive impact after 23 months. Access to improved sanitation was associated with an increase in HAZ in the NFSS data, but not in the larger DHS data, suggesting the association is spurious.

Household economic status and ethnicity have significant independent impact on HAZ scores in the DHS data only after 23 months. Growth is significantly and substantially reduced in the case of Indian Tamils and, to a lesser extent, in Muslims. Household wealth has an independent, direct impact equivalent to an increase of 0.4 HAZ scores between poorest and richest quintiles. This is only a quarter of the difference in observed scores between poorest $(-1.6)$ and richest quintiles (-0.6), indicating that other factors explain most of the inequality.

To assess the relative magnitude of the impacts on HAZ of child feeding practices, household socioeconomic status, birth weight and maternal height, we used the DHS 2006/07 23-59 months model estimates to predict the impact of changing key determinants on predicted HAZ at 59 months. This involved adjusting selected factors to optimal values for all children in the sample, and then predicting the HAZ scores. As shown by the results in Figure 2,

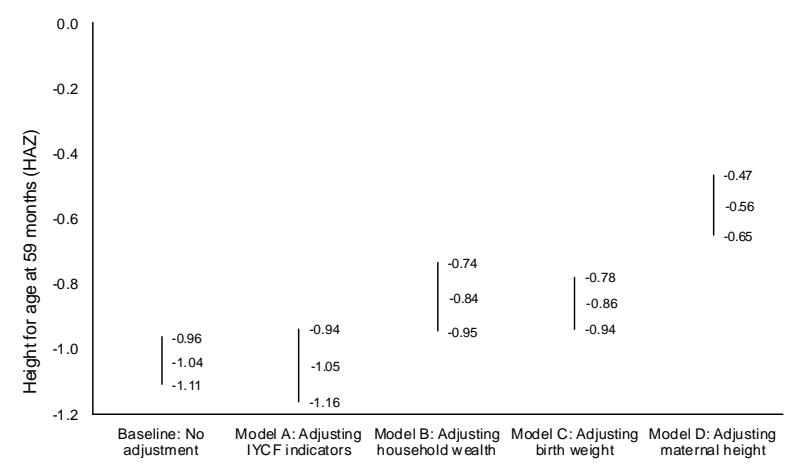

Figure 2. Effect of adjusting for key factors on predicted HAZ scores at 59 months (means with $95 \%$ confidence intervals).

Model A: all modelled IYCF indicators set to optimal values. Model B: household wealth level set to richest quintile for all observations. Model C: Birth weight adjusted to median weight in WHO growth standards (3,290 g). Model D: Maternal height adjusted to mean height in WHO MGRS study $(161.0 \mathrm{~cm})$. improving IYCF indicators to the optimal level for all children has negligible impact, whilst adjusting household wealth or birth weight has significant impacts. However, the largest impact, equivalent to an increase of almost 0.5 $z$ scores, or halving the negative HAZ scores, is seen when maternal height is adjusted to a level of $161.0 \mathrm{~cm}$, which was the mean height of mothers in the multicountry study used to generate the WHO growth standards [11]. Sri Lankan mothers are much shorter than this, with a mean height of $152.4 \mathrm{~cm}$ in the DHS 2006/07.

\section{Discussion}

Our analysis confirms a substantial decline in stunting and underweight in Sri Lanka between the 1980s and 2000s, but no significant decline between 2000 and 2006/07. Differences between the DHS and the NFSS surveys prevent further assessment of the trend to 2009 . This stagnation in the $2000 \mathrm{~s}$ is despite significant increases in average living standards (real per capita GDP increased by $34 \%$ between 1998 and 2005), as well as substantial improvements in infant and child feeding practices, which have been claimed to be critical for improving undernutrition $[12,19,20]$. The degree of socioeconomic inequality in stunting and underweight is also high compared with other countries.

Other than proximate biological determinants, our results indicate that three major factors explain stunting in Sri Lanka: income, low birth weight and maternal height. Differences in child feeding practices and child and maternal illness have little influence, except continued breastfeeding for at least 12 months. The NFSS data do not reveal a significant relationship with food security, but the available variables do not directly assess actual food intake in quantity terms, and the sample was relatively small. On the other hand, the large positive impact of household living standards on HAZ after 23 months and the negative association with maternal underweight, which is strongly correlated with household wealth, suggest that income acts via its influence on access to food. The birth weight results reinforce this, as our previous findings suggest that poverty acts via food insecurity to increase the risk of low birth weight [9]. The impact of food insecurity is manifested initially, almost wholly, in reduced birth weights, and its continuing impact only becomes evident after the first two years of life in slowed growth. This implies that poverty reduction and increased food security provide the greatest potential for reducing stunting rates in Sri Lanka. Research to elaborate the role of food insecurity and its link with poverty in causing undernutrition in Sri Lanka, and to explain why food security has not improved for the poorest Sri Lankans despite strong economic growth in the past decade, should be of highest priority.

This leaves maternal height as the other major determinant of low birth weight, as well as subsequent 
height growth and stunting. The substantial improvement in predicted HAZ when maternal height is adjusted to 161 $\mathrm{cm}$ is noteworthy. We explored this further, by reweighting the DHS 2006/07 data to match the reported distribution of maternal heights in the MGRS study that generated the WHO growth standard (mean=161.0, $\mathrm{SD}=7.2$ ), which is equivalent to picking a sample of Sri Lankan mothers with similar height parameters [11]. In this reweighted sample, not only are HAZ scores substantially higher, but the overall rate of stunting falls to $4 \%$ in the richest quintile (overall $9 \%$, poorest quintile 19\%), implying that in the most affluent families in Sri Lanka, the children already grow at similar rates to the WHO reference population, when maternal heights are similar. This implies that about half the shortfall in child height growth in Sri Lanka, and almost all that in richest quintile, can be explained by the shortness of the average Sri Lankan mother, compared to those in the MGRS study. Since adult heights reflect not only genetic potential but also epigenetic influences from previous generations, and since there is no evidence to indicate differences in genetic potential for height between most human populations, a substantial part of the stunting observed in Sri Lanka must be due to epigenetic factors that are proxied by current adult heights, indicating the importance of intergenerational influences. A similar explanation has been offered in the study of child growth in Hong Kong SAR, a population which is one of the ten most affluent and healthiest in the world, but where mothers remain shorter than in the WHO MGRS study [21]. Our Sri Lankan findings echo this, and suggest that the WHO growth standards may not adequately describe optimal growth patterns in populations where epigenetic factors still substantially constrain adult heights.

\section{Acknowledgements}

We acknowledge financial support from UNICEF, Colombo (Agreement No. SSA/SRIA/2010/00003395-0) and the IHP Public Interest Research Fund (Grant No. PIRF-2011-03). We also thank the Department of Census and Statistics for making available the data from the DHS surveys.

\section{References}

1. Victora CG, Adair L, Fall C, et al. Maternal and child undernutrition: consequences for adult health and human capital. Lancet 2008; 371: 340-57.

2. Department of Census and Statistics (DCS) and Ministry of Health Nutrition and Welfare (MOH). Sri Lanka Demographic and Health Survey 2000. Colombo, Sri Lanka: Department of Census and Statistics in collaboration with Ministry of Health, Nutrition and Welfare, 2002.

3. Department of Census and Statistics (DCS) and Ministry of Healthcare and Nutrition (MOH). Sri Lanka Demographic and Health Survey 2006-07. Colombo, Sri Lanka: Department of Census and Statistics in collaboration with Ministry of Healthcare and Nutrition, 2009.

4. Demographic and Health Survey 1993. Colombo, Sri Lanka: Department of Census and Statistics, Ministry of Finance, Planning, Ethnic Affairs and National Integration in collaboration with Ministry of Health, Highways and Social Science, 1993.

5. Sri Lanka Demographic and Health Survey 1987. Columbia, United States: Westinghouse; Institute for Resource Development 1987.

6. Jayasinghe S. Narrowing health inequalities in Sri Lanka: Issues and challenges. Ceylon Medical Journal 2010; 55: 73-6.

7. Jayatissa R, Hossaine SMM. Nutrition and Food Security Assessment in Sri Lanka 2009. Colombo, Sri Lanka: Medical Research Institute, UNICEF and World Food Programme, 2010.

8. Filmer D, Pritchett L. Estimating wealth effects without income data or expenditure data - or tears: Educational enrollment in India. Demography 2001; 38: 115-32.

9. Anuranga C, Wickramasinghe R, Rannan-Eliya RP, Hossain SM, Abeykoon A. Trends, inequalities and determinants of low birth weight in Sri Lanka. Ceylon Medical Journal 2012; 57: 61-9.

10. National Center for Health Statistics. NCHS Growth Curves for Children, Birth to 18 Years. Washington, DC: National Center for Health Statistics, Department of Health, Education, and Welfare; 1977.

11. WHO Multicentre Growth Reference Study Group. WHO child growth standards based on length/height, weight and age. Acta Paediatrica Supplement 2006; 450: 76-85.

12. Victora CG, de Onis M, Hallal PC, Blossner M, Shrimpton R. Worldwide timing of growth faltering: revisiting implications for interventions. Pediatrics 2010; 125: 473-80.

13. Anuranga C, Wickramasinghe R, Rannan-Eliya RP, Hossain SM, Jayatissa R, Abeykoon A. Trends and inequalities in child undernutrition, Sri Lanka 1987-2009. IHP Health Statistics Reports, No 3. Colombo: Institute for Health Policy (In Press).

14. Van de Poel E, Hosseinpoor AR, Speybroeck N, Van Ourtia $\mathrm{T}$, Vega J. Socioeconomic inequality in malnutrition in developing countries. Bulletin of the World Health Organization 2008; 86: 282-91.

15. Wagstaff A, Paci P, van Doorslaer E. On the measurement of inequalities in health. Social Science and Medicine 1991; 33: 545-57.

16. Indicators for assessing infant and young child feeding practices: conclusions of a consensus meeting held 6-8 November 2007. Geneva, Switzerland: World Health Organization, 2008.

17. Strategy for Improved Nutrition of Children and Women in Developing Countries. New York: UNICEF, 1990.

18. Jensen GM, Moore LG. The Effect of High Altitude and Other Risk Factors on Birthweight: Independent or 
Interactive Effects? American Journal of Public Health 1997; 87: 1003-7.

19. Senarath U, Siriwardena I, Godakandage SSP, Jayawickrama H, Fernando DN, Dibley MJ. Determinants of breastfeeding practices: An analysis of the Sri Lanka Demographic and Health Survey 2006-2007. Maternal and Child Nutrition 2011; 8: 315-29. doi: 10.1111/j.740-8709.2011.00321.x.
20. Shekar M, Somanathan A, Du L. Malnutrition in Sri Lanka: Scale Scope, Causes and Potential Response. Washington, DC: World Bank, 2007.

21. Hui LL, Schooling CM, Cowling BJ, Leung SSL, Lam TH, Leung GM. Are universal standards for optimal infant growth appropriate? Evidence from a Hong Kong Chinese birth cohort. Archives of Disease in Childhood 2007; 93: 561-5.

\title{
Obstetric performance of women who have previously delivered a baby of low birthweight
}

\author{
H Senanayake, S Bujawansa, V Kariyawasam, H Ariyaratne \\ (Index words: previous term low birth weight, obstetric risk factors, pregnancy outcomes)
}

\begin{abstract}
Objectives To investigate pregnancy outcomes in women who have previously given birth to a baby weighing less than 2500 grams in a population in which the low birthweight rate is high.

Methods We compared the obstetric performance of 100 women who had delivered a low birthweight baby $(<2500 \mathrm{~g})$ at term in a previous pregnancy (cases) with those of 100 women who were matched for age, height, body mass index at booking, parity and medical disorders, who had previously delivered a baby above that threshold (controls).
\end{abstract}

Results We found the following significant differences between cases and controls ( $p<0.001$ for all). Gestation at delivery (mean $\pm \mathrm{SD}=38.0 \pm 1.9$ vs. $39.1 \pm 1.2$ weeks); risk of delivery before completion of 37 weeks (19.0\% vs. $3.0 \%$ ); birthweight (mean $\pm \mathrm{SD}=2.553 \pm 0.444$ vs. $3.015 \pm 0.413 \mathrm{Kg}$ ); risk of delivery of a low birthweight (LBW) baby in the current pregnancy (27.0\% vs. $4.0 \%)$. The stillbirth rate was also significantly higher among cases (4 vs. $0 ; p<0.05$ ).

Conclusions Birth of a LBW baby points to adverse events in future pregnancies even where its prevalence is high. It is a useful criterion for assessment of risk in antenatal care and for directed interventions.

Ceylon Medical Journal 2013; 58: 18-21

\section{Introduction}

Low birth weight (LBW) babies (birth weight $<2.5 \mathrm{~kg}$ ) are known to have a poor perinatal outcome and to make a disproportionate contribution to perinatal deaths $[1,2]$. In addition, they are prone to important long term complications [3].

LBW babies born at term could be considered as being small for gestational age (SGA) irrespective of ethnicity and maternal body mass index (BMI). There are a multitude of immediate and inter-generational risk factors that underlie the birth of a baby who weighs less than 2.5 $\mathrm{kg}$ at term. These include short maternal height, low maternal BMI, poor weight gain during pregnancy, maternal race, medical disorders complicating pregnancy (e.g. hypertension), substance abuse and a previous low birthweight baby. Many studies have addressed these factors, but there is little quality evidence on the future reproductive performance of women who have previously delivered LBW babies.

The majority of risk factors for LBW are likely to persist into future pregnancies and these women could potentially be at higher risk of a repeat birth of a LBW baby. Risk factors for LBW are also common to other adverse pregnancy outcomes and it is possible that the birth of a LBW baby may point to other adverse outcomes in future pregnancies. It could therefore serve as a risk factor to screen women during pregnancy.

Two previous studies have shown that women who delivered LBW babies have a greater tendency to repeat a LBW birth. However, both these studies were not restricted

Department of Obstetrics and Gynaecology, Faculty of Medicine, University of Colombo, Sri Lanka.

Correspondence: HS, e-mail: <senanayakeh@gmail.com>. Received 4 May and revised version accepted 13 October 2012. Competing interests: none declared. 